\title{
Memoria Colectiva e Historia Universal: Evaluación Subjetiva e Importancia de Eventos y Personajes
}

\author{
Gisela Isabel Delfino ${ }^{1}$, Fernanda Mariel Sosa ${ }^{2}$, Magdalena Bobowik ${ }^{3}$, Elena Mercedes \\ Zubieta ${ }^{4}$
}

\section{RESUMEN}

La memoria colectiva hace referencia a cómo los grupos sociales recuerdan, olvidan o se reapropian del conocimiento del pasado social. Siguiendo los estudios previos que toman a la historia como objeto de representación social, se analiza cómo los eventos y personajes de la historia universal se agrupan en función de su evaluación e importancia $(n=379,55.7 \%$ mujeres, edad $=18-69)$. Los datos fueron recolectados a través de internet utilizando un cuestionario autoadministrado. Se presentaron 28 eventos y 24 personajes de la historia universal frente a los cuales los participantes debian indicar en qué medida les parecían positivos o negativos y qué importancia les atribuian. Se efectuaron dos análisis de conglomerado jerárquico de variables, uno para evaluación subjetiva y otro para importancia. Para la evaluación subjetiva se obtuvieron tres conglomerados y para importancia se hallaron dos conglomerados. Los estimulos evaluados de forma más negativa pertenecen al último siglo y están relacionados con guerras o problemas politicos y económicos. Aquellos evaluados de forma más favorable se asocian con descubrimientos o con la lucha contra la opresión (abolición de la esclavitud, sistema democrático). En cuanto a la importancia atribuida, todos los estímulos presentados obtuvieron una alta importancia, alcanzando los eventos puntuaciones más elevadas que los personajes. Los eventos y personajes considerados más importantes se relacionaron con avances en los derechos del hombre y la libertad, el conocimiento y cambios en la vida social. Estos hallazgos se discuten en el marco de la memoria colectiva.

Palabras clave: memoria, historia, grupos sociales, percepción social.

\section{Collective Memory and Universal History: Subjective Evaluation and Importance of Events and Historical Characters}

\begin{abstract}
Collective memory refers to how social groups remember, forget or re-appropriate knowledge of the social past. Following previous studies that take history as an object of social representation, it is analyzed how events and characters of universal history are grouped together according to their evaluation and importance $(n=379,55.7 \%$ women, age $=18-69$ ). Data was collected through the internet using a self-administered

1 Pontificia Universidad Católica Argentina, Consejo Nacional de Investigaciones Científicas y Técnicas (CONICET), Argentina; gisela_delfino@uca.edu.ar; http://orcid.org/0000-0002-3732-184X

2 Universidad de Buenos Aires, Consejo Nacional de Investigaciones Científicas y Técnicas (CONICET), Argentina; fernandamarielsosa@hotmail.com; http://orcid.org/0000-0003-1445-1710

3 Universidad Pompeu Fabra, España; magdalena.bobowik@upf.edu; http://orcid.org/0000-0002-23410665

4 Universidad de Buenos Aires, Consejo Nacional de Investigaciones Científicas y Técnicas, Argentina; elenazubieta@hotmail.com; http://orcid.org/0000-0002-8789-737X
\end{abstract}

NOTA DEL AUTOR | Esta investigación se ha realizado gracias al financiamiento PIP11220130100313CO otorgado por CONICET. La correspondencia relativa a este artículo debe ser dirigida a Gisela Isabel Delfino, e-mail: gisela_delfino@uca.edu.ar 
questionnaire. Twenty-eight events and 24 characters from world history were presented, and participants had to indicate to what extent they seemed positive or negative and what importance they attributed to them. Two hierarchical cluster analyzes were performed, one for subjective evaluation and one for importance. For the subjective evaluation three clusters were obtained and for importance two clusters were found. The stimuli evaluated in the most negative way belong to the last century and are related to wars or political and economic problems. Those evaluated most favorably are associated with discoveries or with the fight against oppression (abolition of slavery, democratic system). Regarding the importance attributed, all the stimuli presented obtained a high importance, reaching events higher scores than characters. Events and characters considered most important were related to advances in human rights and freedom, knowledge and changes in social life. These findings are discussed within the framework of collective memory.

Keywords: social, history, social groups, social perception.

La psicologia ha estudiado tradicionalmente la memoria desde un modelo estático e individualista, reforzando la idea de su exactitud y su poder evocativo. Sin embargo, durante la última tercera parte del último siglo la memoria es entendida como una actividad constructiva, cuestionándose la precisión respecto del pasado en su actualización en el presente. Los cambios producidos en los estudios sobre la memoria muestran un acuerdo creciente con la idea de la memoria como una actividad social que se realiza no en privado sino a través de la conciencia social compartida con los otros (Valencia, Momoitio, \& Idoyaga, 2010). Esta perspectiva de la memoria está asociada a la idea de Halbwachs (1925/2004), quien, siguiendo el abordaje de Durkheim, sostiene que la memoria solo es posible a través de la participación en la vida social (Wagner, 2015). Específicamente, define a la memoria colectiva (MC) como el conjunto de representaciones sobre el pasado que un grupo produce, conserva, elabora y transmite mediante la interacción de sus miembros. La MC se refiere a cómo los grupos sociales recuerdan, olvidan o se reapropian del conocimiento del pasado social. No se trata solo de las huellas que el pasado deja en el presente de las personas, sino de lo que se ha producido precisamente para ser transmitido a generaciones sucesivas (Páez, Techio, Marques, \& Beristain, 2007). La actividad reconstructiva de la memoria tiene un limite que reside en la pluralidad de memorias colectivas que se hacen presentes en una sociedad, así como en la misma historiografia (Rosa, Belleli, \& Bakhurst, 2000).

Las memorias colectivas no son solo representaciones, también son actitudes cognitivas y prácticas afectivas que prolongan de manera irreflexiva las experiencias pasadas en el presente (Valencia \& Páez, 1999). Estas prácticas sociales que: "vinculan el pasado y el presente, son costrumbres operativas, cognitivas y relaciones que constituyen el tejido de la continuidad de cada grupo social, entrelazado con un universo de significados, de valores y narraciones que la dotan de cierto automatismo" (Páez et al., 2007, p. 694).

La memoria se apoya en el procesamiento interpersonal de la información, que está distribuida entre los miembros del grupo y, por lo tanto, es más que la suma de recuerdos individuales. Objetos semióticos e instituciones sirven de mediación y apoyo externo al recuerdo y olvido, y hay efectos globales del recuerdo y del olvido que no dependen directamente de actividades individuales conscientes y voluntarias (Páez, Valencia, Pennebaker, Rime \& Jodelet, 1998). 
Según Halbwachs (1968/1995), la memoria es social por sus contenidos. Se recuerda un mundo en el que vive también otra gente por lo que la memoria del pasado es una memoria de un pasado intersubjetivo, vivido con otras personas. Se apoya en marcos sociales de referencia, como los rituales, las ceremonias y los eventos sociales. Las personas tienen memorias compartidas y recordadas conjuntamente que se basan en el lenguaje y la comunicación lingüística externa e interna con otros significativos. Además, la memoria es social por sus funciones: ser miembro de un grupo implica compartir la MC de este, asumir e internalizar tradiciones comunes y representaciones sociales. El recuerdo permite mantener una visión positiva del grupo. Sin embargo, no se puede afirmar que haya una arbitrariedad y libertad absoluta en la elección del contenido de la MC (Páez et al., 2007).

Tomando la memoria como una actividad social que, si bien reside en los individuos, se realiza a través de la conciencia social compartida con otros y que se inserta en dinámicas funcionales e intergrupales complejas, la historia se convierte en un objeto de estudio con caracteristicas similares a las planteadas por la teoría de las representaciones sociales (Haas \& Jodelet, 2000). La historia define una trayectoria que ayuda a construir la esencia de la identidad grupal y la relación de esta con otros grupos. Provee, además, de una narrativa que les dice a los miembros de un grupo quiénes son, de dónde vienen y a dónde van, justificando las respuestas y actitudes hacia los desafios actuales (Hilton \& Liu, 2017; Liu \& Hilton, 2005; Roth, Huber, Juenger, \& Liu, 2017).

Las representaciones sociales de la historia (RSH), como construcciones sociales del conocimiento de sentido común, guían a los individuos en el contexto inmediato, permitiendo su adaptación al mundo físico y su integración en las interacciones sociales, cuestionando la pertenencia a diferentes grupos. Actúan como mito fundacional para una sociedad, definiendo sus derechos y obligaciones, legitimando sus acuerdos políticos y adjudicando roles en función de lo que es correcto o incorrecto hacer apelando a la experiencia histórica (Sibley, Liu, Duckitt, \& Khan, 2008). Como señalan Liu y Hilton (2005), las RSH recurren a la historia como un reservorio simbólico que contiene relatos de acontecimientos y personajes cuya relevancia emocional es ampliamente compartida, y al que los individuos apelan para justificar posiciones, comportamientos y la forma de resolver conflictos. La historia constituye la materia prima que se comunica a través de canales interpersonales e institucionales para producir representaciones simbólicas que consisten en narraciones e imágenes icónicas que mantienen la continuidad entre el pasado, el presente y el futuro de un pueblo (Liu et at., 2012). Estas representaciones sociales del pasado tienen su relevancia en la medida en que las memorias colectivas demuestran ejercer impacto sobre las actitudes frente a nuevos hechos (Páez et al., 2007).

Las RSH contienen componentes descriptivos como eventos y personajes importantes enlazados en historias con formas temporales referidas a mitos de origen. Sibley et al. (2008) indican que la secuencia temporal del recuerdo sigue un patrón en el que los eventos fundacionales y los recientes son los nombrados con mayor frecuencia en comparación con los eventos intermedios. Así, la historia se concretiza a través de personajes y eventos ampliamente reconocidos por el grupo y se legitima otorgando temporalidad a través de la imposición de una estructura narrativa. 
En lo que hace al recuerdo y evocación de eventos y personajes de la historia, la literatura (e.g. Liu et al., 2005; Liu et al., 2009) muestra un importante consenso a través de las distintas culturas, donde se destacan: la referencia al pasado reciente, en donde los eventos y figuras de los últimos 100 años son los más evocados, y la focalización en la política y las guerras, con especial protagonismo de la Segunda Guerra Mundial y Hitler como el personaje más influyente. Tanto para los eventos como para los personajes, los estudios (Hanke et al., 2015; Liu et al., 2012) plantean una discrepancia entre el significado o interpretación dada por las sociedades occidentales y no occidentales. Esto es, si bien los eventos y personajes evocados pueden ser similares en distintas culturas, la forma en la que se los evalúa es distinta. No obstante, podría hablarse de un significado occidental compartido y de unas RSH hegemónicas caracterizadas por la centralidad de la historia europea y los eventos culturales occidentales, junto a cierto nacionalismo $\mathrm{o}$ etnocentrismo nacional (Bobowik et al., 2010; Techio et al., 2010).

La evocación a revoluciones y guerras da cuenta del dominio del patrón narrativo de la violencia como partera de la historia o sesgo bélico. A su vez, la definición de la importancia histórica es sumamente egocéntrica, por lo que cada país tiende a incluir eventos históricos nacionales relevantes como más importantes, confirmando el sesgo socio-céntrico. Hay también una tendencia clara a que los eventos recientes se perciban de forma menos positiva que los eventos que ocupan un lugar más lejano en la historia. Se observa un sesgo nostálgico en tanto esfuerzo hacia un sentido positivo y una minimización social de los eventos negativos. La necesidad de mantener una identidad positiva colectiva e individual explica la búsqueda de un significado y el esfuerzo por lograr una reinterpretación positiva (Páez et al., 2007; Zaromb et al., 2018).

Junto a la verificación de sesgos y hegemonías narrativas, las investigaciones más recientes en la perspectiva de las RSH han avanzado en el análisis de los eventos y las figuras evocadas, en posibles clasificaciones y en su evaluación e importancia. Hanke et al. (2015) señalan que los eventos y las figuras de la historia cumplen funciones distintas en la cultura politica de las naciones. Las figuras simbolizan y encarnan valores, mientras que eventos claves como la Segunda Guerra Mundial constituyen esquemas culturales que pueden ser invocados o movilizados como lecciones para justificar la acción (cf. Sosa, Natapof, \& Zubieta, 2017).

En relación con los sucesos de la historia universal que las personas evocan, y sobre la base de muestras de treinta países distintos, Liu et al. (2012) obtienen una clasificación de tres grupos o conglomerados. Estos son, (a) Calamidades históricas: se trata del conglomerado con mayor consistencia y fuerza a lo largo de las distintas culturas e incluye tanto eventos realizados por el hombre como por la naturaleza, intencionales como no intencionales, abarcando las catástrofes de las guerras, los colapsos económicos, los genocidios y el terrorismo; (b) Progreso histórico: incluye eventos tecnológicos y políticos relacionados con el avance de la civilización en tiempos recientes; (c) Resistencia a la opresión histórica o también conceptualizada como lucha por los derechos humanos. Los autores señalan que mientras hay un acuerdo general a lo largo de las culturas respecto de qué eventos constituyen calamidades históricas, es menos claro qué constituye progreso histórico. Explican también que los eventos incluidos en el agrupamiento de progreso histórico son principalmente de origen occidental y que es comprensible que 
sociedades no occidentales tengan opiniones distintas. De forma similar, la resistencia a la opresión parece ser un concepto compartido en las sociedades occidentales, pero no en las no occidentales.

En lo que hace a los personajes o figuras de la historia universal evocados por las personas, Hanke et al. (2015) encuentran, al comparar 35 sociedades, que los científicos, junto con las figuras religiosas y humanitarias asociadas a la paz y habitualmente no tocadas por la polémica politica, no generan prácticamente controversia a lo largo de las culturas y son las más admiradas. Los autores los denominan como "héroes" y "villanos" por la evaluación subjetiva negativa o positiva que los participantes efectuan. Hussein, Bin Laden y Stalin son personajes que generan diferencias entre las distintas muestras, mientras que se observa un consenso respecto de la figura de Hitler, ya que en todas las sociedades analizadas aparece como el personaje peor evaluado, convirtiéndose en el mayor de los villanos.

El tiempo incide en la evaluación de los eventos y las figuras de la historia universal, aunque puede advertirse cierta diferencia del optimismo o la nostalgia. Hanke et al. (2015) observan que figuras antiguas que podrian ser evaluadas de forma negativa (villanos) tienden a recibir evaluaciones neutras. Tal es el caso de personajes como Genghis Khan, Saladin, Qin Emperor y Napoleón. En relación con los eventos, Techio et al. (2010) hallan una evaluación positiva a largo plazo, donde eventos políticos distantes, como la Revolución Francesa o Americana fueron evaluados más positivamente que eventos similares del siglo XX. Puede pensarse que, cuando se trata de eventos, la fuerza del optimismo es mayor y se tiende a hacer una evaluación positiva de los sucesos lejanos, mientras que en los personajes el paso del tiempo tiene una fuerza menor y lleva a una evaluación más neutra. Es importante señalar, de todos modos, que en el caso del estudio sobre personajes de Hanke et al. (2015), tres de los cuatro señalados como ejemplo de personajes evaluados de manera neutra no pertenecen al contexto occidental, de forma que podría estar primando el desconocimiento, una noactitud. Con todo, siguiendo la teoría de la identidad social (Tajfel \& Turner, 2004), puede argumentarse que un colectivo, si quiere seguir siéndolo, tiene que negociar su memoria colectiva: qué recordar, qué olvidar y cómo negociar lo que resulta glorioso o vergonzoso para todos sus componentes, o para algunos de ellos. Como se dijo, se recuerda lo que es adecuado para las actitudes y necesidades grupales actuales, el recuerdo es un instrumento de mantenimiento de una visión positiva del grupo, y las figuras simbolizan y encarnan valores.

Estudios previos muestran que la Segunda Guerra Mundial es uno de los eventos más importantes en más de 30 naciones y también uno de los peor evaluados. Lo mismo ocurre con la figura de Hitler (Bobowik, et al., 2010; Liu et al., 2005; Liu et al., 2012; Pennebaker, Páez, \& Deschamps, 2006). Estos datos se corroboran en el contexto argentino a la hora de indagar en evocaciones sobre la historia universal (Bombelli, Sosa, Fernández, Cejas, \& Torres, 2013; Cejas, Fernández, \& Sosa, 2013; Sosa, Bombelli, Cejas, \& Fernández, 2013).

Los estudios en RSH han analizado mayormente la evocación (Liu et al., 2005; Liu et al., 2009), la evaluación (Bobowik et al., 2010; Hanke et al., 2015; Liu et al., 2009; Liu et al., 2012, Techio et al., 2010) o la importancia atribuida (Techio et al., 2010) a distintos eventos y personajes de la historia. Sin embargo, no se conocen estudios que hayan explorado la relación entre la 
evaluación y la importancia atribuida. Además de plantear conocer cómo evalúa y la importancia que le atribuye una muestra de población de la zona metropolitana de Buenos Aires (Argentina) a los eventos y personajes de la historia de manera conjunta, se busca explorar la relación entre el sentido de la evaluación (positivo-negativo) y la importancia (nada-extrema) atribuida a los eventos y personajes de la historia universal, enmarcando el análisis en las agrupaciones propuestas por Liu et al. (2012) y Hanke et al. (2015), respectivamente, así como en los sesgos verificados en estudios previos.

\section{MÉTODO}

\section{Diseño} transversal.

El estudio es de naturaleza descriptivo correlacional de diseño

\section{Participantes}

La muestra no probabilística intencional estuvo compuesta por 379 participantes residentes en la ciudad de Buenos Aires y el conurbano bonaerense. Del total de los encuestados, un $55.7 \%$ eran mujeres y un $44.3 \%$ eran hombres. La media de edad fue de $36.20(D E=11.17)$, siendo el rango de edad 18-69 y la mediana igual a 34 años. En lo que hace al estado civil, un $36.7 \%$ era soltero/a, un $23 \%$ convivia o estaba en pareja de hecho, un $32.7 \%$ estaba casado/a, un $6.6 \%$ divorciado/a o separado/a y un $1.1 \%$ era viudo/a. Del total de los consultados, el 77\% trabajaba y de estos un $65.5 \%$ lo hacía en el sector privado. Asimismo, el $23.7 \%$ de la muestra estudiaba en la universidad, de los que un $67 \%$ concurría a una universidad pública y un $53.8 \%$ estudiaba carreras relacionadas con las ciencias sociales o las humanidades.

\section{Instrumentos}

El cuestionario utilizado fue de formato autoadministrado y estuvo integrado por distintas preguntas y secciones de la cuales aquí solo se reportan dos, una relativa a eventos y otra referente a personajes de la historia universal. Se trabajó con 28 eventos y 24 personajes de la historia universal (ver tabla 1). El listado de los eventos y personajes se confeccionó sobre la base del estudio de Liu et al. (2005), en el que se analizan los eventos y personajes más importantes para doce culturas, y a los hallazgos obtenidos en un estudio piloto previo en el que se solicitó a 60 participantes la mención de eventos y personajes.

Se pidió a los participantes que indiquen en qué medida les parecían positivos o negativos estos eventos y personajes. La opción de respuesta fue de 1 (extremadamente negativo) a 7 (extremadamente positivo). También se les solicitó que señalen qué importancia les atribuian a los mismos eventos y personajes sobre la base de una opción de respuesta de 1 (nada importante) a 7 (extremadamente importante).

\section{Procedimiento}

Los datos fueron recolectados a través de Internet, enviando un e-mail a conocidos, que a su vez contactaron a otros conocidos (método bola de nieve), donde se proporcionaba un link desde el cual se accedia al cuestionario on- 
line. La participación fue voluntaria y anónima, y el único criterio de inclusión fue que los participantes fueran mayores de 18 años y residentes de la zona metropolitana de Buenos Aires. Las respuestas fueron recabadas durante un lapso de 25 dias. Antes de comenzar con la encuesta, los participantes debian firmar un consentimiento informado, que cumple con los lineamientos para el comportamiento ético en las Ciencias Sociales y Humanas establecidos por el Consejo Nacional de Investigaciones Científicas y Técnicas (CONICET) de Argentina (Res. D N $\mathrm{N}^{\circ}$ 2857/06), en el que se les informaba acerca de los propósitos de la investigación.

\section{Análisis de datos}

Se efectuaron análisis descriptivos para cada evento y personaje de la historia universal y se realizó un análisis de conglomerado jerárquico de variables (método vinculación inter-grupos, distancia euclídea al cuadrado) tanto para la evaluación en la dimensión positivo-negativo como para la atribución de importancia, incluyendo eventos y personajes. Para establecer la relación entre evaluación en la dimensión positivo-negativo y la importancia atribuida a los eventos y personajes tomados en general, se confeccionó una tabla de contingencia y se calculó el Chi Cuadrado. Asimismo, se efectuaron comparaciones entre las medias en las evaluaciones de eventos y personajes ( $t$ de Student) y correlaciones ( $r$ de Pearson).

\section{RESULTADOS}

\section{Evaluación en la dimensión positivo-negativa de los eventos y personajes}

El promedio obtenido en la evaluación de los 28 eventos $(M=4.18, D E=$ $0.51)$ coincide con el promedio hallado para los 24 personajes $(M=4.18, D E=$ $0.53)$. Teniendo en cuenta que la media teórica es 4 , se verifica una evaluación total intermedia, con un pequeño sesgo hacia lo positivo. El análisis de rango muestra valores levemente más positivos para los eventos que para los personajes (Eventos: Mín = 2.07; Máx = 6.46; Personajes: Mín $=1.92 ;$ Máx $=$ $5.33)$, sin hallarse diferencia al comparar las medias, $t(298)=-0.438 ; p=.662$; 95\% IC [-0.07; 0.04].

La tabla 1 exhibe las medias de evaluación subjetiva obtenidas para cada uno de los eventos y personajes de la historia universal. En la figura 1 se muestra el dendrograma obtenido del análisis de conglomerado jerárquico de variables. 
Tabla 1.

Evaluación subjetiva evocada por los eventos y personajes de la historia universal. Medias y desviaciones estándar (de más negativo a más positivo).

\begin{tabular}{|c|c|c|c|c|c|}
\hline EVENTOS & $M$ & $D E$ & PERSONAJES & $M$ & $D E$ \\
\hline Holocausto & 1.22 & 0.77 & Hitler & 1.22 & 0.72 \\
\hline Bombas atómicas & 1.24 & 0.85 & Thatcher & 1.64 & 1.11 \\
\hline Nazismo & 1.35 & 0.99 & Bin Laden & 1.75 & 1.17 \\
\hline Desastres naturales & 1.61 & 1.29 & Mussolini & 1.87 & 1.18 \\
\hline I Guerra Mundial & 1.73 & 1.27 & Bush & 1.95 & 1.13 \\
\hline II Guerra Mundial & 1.74 & 1.41 & Stalin & 2.58 & 1.41 \\
\hline Guerra de Irak (2005) & 1.75 & 1.37 & Fidel Castro & 3.55 & 1.88 \\
\hline Guerra Fría & 1.94 & 1.21 & Napoleón & 3.64 & 1.51 \\
\hline Atentado del 11/09 & 2.07 & 1.52 & Churchill & 3.76 & 1.50 \\
\hline Gran depresión & 2.56 & 1.32 & Kennedy & 4.04 & 1.41 \\
\hline Revolución Rusa & 3.57 & 1.63 & Cristóbal Colón & 4.04 & 1.80 \\
\hline Imperio Romano & 3.97 & 1.50 & Martín Lutero & 4.19 & 1.34 \\
\hline Revolución Cubana & 4.11 & 1.84 & Alejandro Magno & 4.31 & 1.47 \\
\hline Globalización & 4.13 & 1.65 & Che Guevara & 4.45 & 1.99 \\
\hline Descubrimiento de América & 4.26 & 2.08 & Juan Pablo II & 5.10 & 1.78 \\
\hline Llegada hombre luna & 4.93 & 1.55 & Freud & 5.26 & 1.55 \\
\hline Revolución Francesa & 4.94 & 1.56 & Aristóteles & 5.32 & 1.42 \\
\hline Revolución Industrial & 5.04 & 1.51 & Jesús & 5.43 & 1.72 \\
\hline Nacimiento de Jesús & 5.29 & 1.77 & Luther King & 5.72 & 1.46 \\
\hline Caída Muro Berlín & 5.48 & 1.66 & Da Vinci & 5.95 & 1.27 \\
\hline Era digital & 5.60 & 1.45 & Mandela & 6.03 & 1.44 \\
\hline Evolución de la humanidad & 5.74 & 1.42 & Einstein & 6.13 & 1.13 \\
\hline Invención imprenta & 5.91 & 1.40 & Teresa de Calcuta & 6.13 & 1.32 \\
\hline Desarrollos tecnológicos & 5.91 & 1.23 & Mahatma Gandhi & 6.21 & 1.19 \\
\hline Independencia colonias & 5.94 & 1.39 & & & \\
\hline Sistema democrático & 6.00 & 1.29 & & & \\
\hline Abolición esclavitud & 6.42 & 1.36 & & & \\
\hline $\begin{array}{l}\text { Descubrimientos } \\
\text { médicos/científicos }\end{array}$ & 6.43 & 1.02 & & & \\
\hline
\end{tabular}

En el dendrograma (figura 1) se observan de manera clara 3 conglomerados. El primer conglomerado incluye 10 eventos y 6 personajes que presentan evaluaciones promedio negativas, todas menores a 2.58. Todos los eventos y personajes evaluados de forma negativa pertenecen al siglo XX o XXI e involucran, al menos en parte, a Estados Unidos y a Europa y están relacionados a guerras o, en su defecto, a problemas políticos y económicos. Si bien los desastres naturales podrian pensarse como presentes en distintos momentos de la historia universal, la Base de datos internacional sobre desastres (Laframboise \& Acevedo, 2014) señala un mayor registro de estos en las últimas décadas. Se observa en este grupo a las catástrofes sociales y a los villanos. 

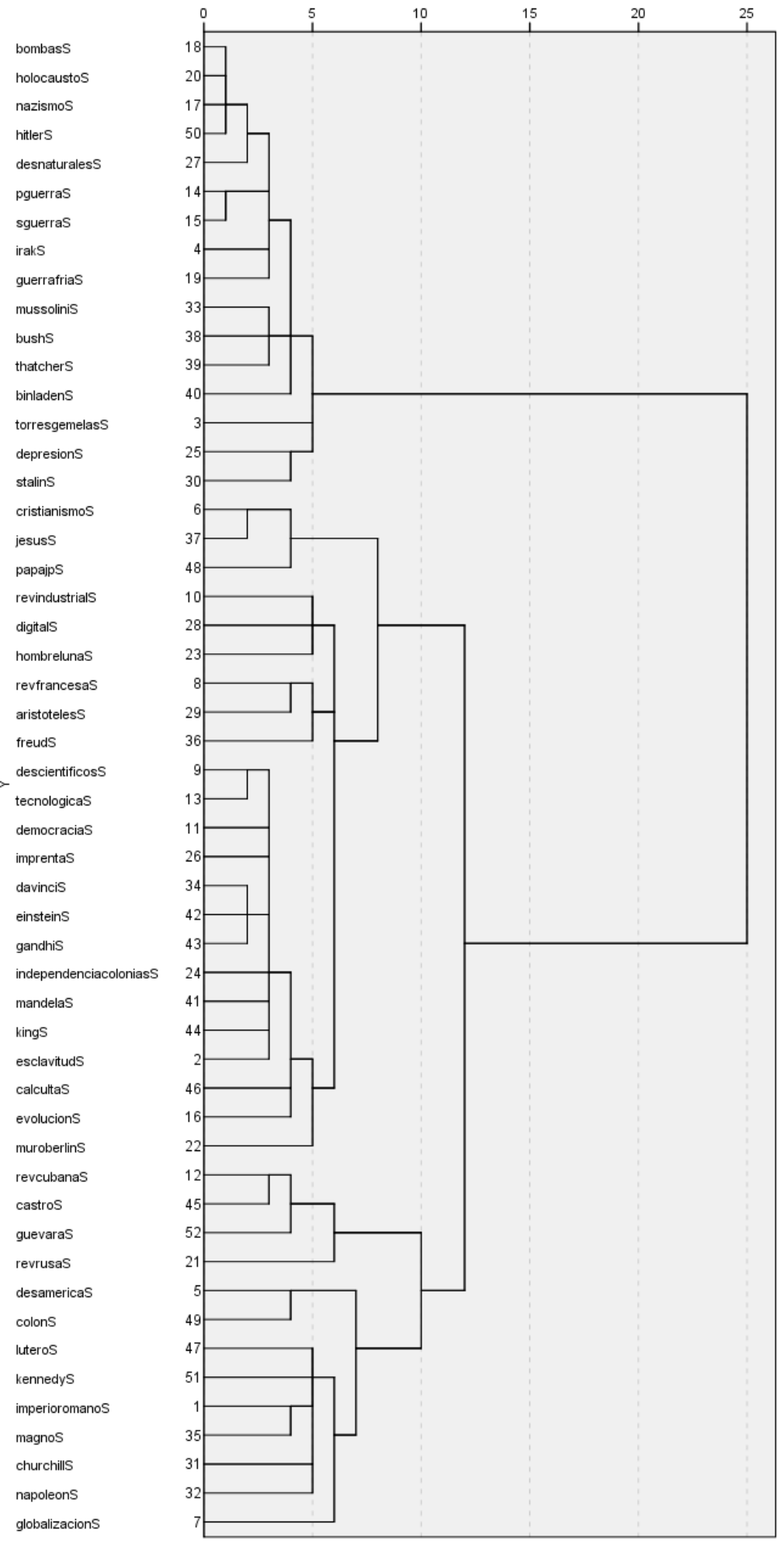

Figura 1. Dendrograma para evaluación subjetiva evocada por los eventos y personajes presentados.

El segundo conglomerado, ubicado espacialmente en el centro del dendrograma y que se extiende desde el Nacimiento de Jesús de Nazaret (cristianismo) hasta la caída del Muro de Berlin, incluye 13 eventos y 10 personajes evaluados positivamente, todos con medias mayores a 4.93. Entre los eventos y personajes de este conglomerado no se detectan guerras ni 
personajes relativos a ellas, aunque si aparecen hechos que implicaron violencia (Revolución Francesa e independencia de las colonias). Se observan líderes políticos y sociales evaluados positivamente, que se caracterizan por su preocupación por cuestiones humanitarias y de paz, así como personajes relacionados con logros científicos y con las artes. La ausencia de sesgo bélico -amortiguado en revolución francesa por sesgo nostálgico- y de personajes asociados con la violencia, hace surgir en este grupo al progreso social y a los héroes.

El tercer conglomerado, ubicado en la parte inferior del dendrograma, se inicia con la Revolución Cubana e incluye 5 eventos y 8 personajes con evaluaciones entre 3.55 y 4.45. Si bien la mayoría de los eventos y personajes refieren a Estados Unidos y a Europa, se hace clara la presencia del continente americano en general, y aunque siguen prevaleciendo las guerras, hay una orientación más a luchas sociales y a cuestiones relativas a conquistas y exploraciones. Es una agrupación intermedia en la que sobresale el socio-centrismo.

En el dendrograma se corrobora también una relación temática entre eventos y personajes. En el conglomerado negativo se observa: (a) las bombas atómicas, el Holocausto, el nazismo y Adolf Hitler; (b) la Primera y la Segunda Guerra Mundial; (c) Benito Mussolini, George W. Bush y Margaret Thatcher. En el conglomerado positivo: (a) el nacimiento de Jesús de Nazaret (Cristianismo), Jesús de Nazaret y el Papa Juan Pablo II; (b) la Revolución Industrial, la era digital, la llegada del hombre a la Luna, los desarrollos tecnológicos y los descubrimientos médicos/científicos; (d) Leonardo da Vinci, Albert Einstein y Mahatma Gandhi. Y en el tercer conglomerado, más sociocéntrico y de carácter intermedio por sus evaluaciones, se detecta: (a) la Revolución Cubana, Fidel Castro y Ernesto Che Guevara; y (b) el descubrimiento de América y Cristóbal Colón.

\section{Importancia atribuida a los eventos y personajes}

La media de importancia para los 28 eventos es de $5.68(D E=0.79)$ y para los 24 personajes es de $5.11(D E=0.95)$, indicando que la importancia total atribuida a los estímulos presentados es en general alta. El análisis de rango muestra valores más elevados para los eventos que para los personajes (Eventos: Mín $=3.14 ;$ Máx $=7 ;$ Personajes: Mín $=1.92 ;$ Máx $=7$ ), confirmándose esta diferencia al comparar las medias con un tamaño del efecto mediano, $t(309)=15.997 ; p<.001 ; g=0.62 ; 95 \%$ IC $[0.46 ; 0.60]$.

La tabla 2 presenta el promedio de importancia atribuida a cada uno de los eventos y personajes de la historia universal y la figura 2 exhibe el dendrograma producto del análisis de conglomerado jerárquico de variables. 
Tabla 2.

Importancia atribuida a los eventos y personajes de la historia universal. Medias y desviaciones típicas (menos importante a más).

\begin{tabular}{|c|c|c|c|c|c|}
\hline EVENTOS & $M$ & $D E$ & PERSONAJES & $M$ & $D E$ \\
\hline Revolución Cubana & 4.69 & 1.73 & Bush & 3.71 & 1.87 \\
\hline Revolución Rusa & 4.70 & 1.65 & Thatcher & 3.87 & 2.07 \\
\hline Guerra Fría & 4.80 & 1.80 & Bin Laden & 4.01 & 2.10 \\
\hline Llegada del hombre a la Luna & 4.84 & 1.82 & Mussolini & 4.35 & 1.77 \\
\hline Gran depresión & 4.86 & 1.59 & Kennedy & 4.46 & 1.62 \\
\hline Guerra de Irak (2005) & 4.99 & 1.71 & Churchill & 4.51 & 1.55 \\
\hline Nazismo & 5.19 & 1.99 & Stalin & 4.65 & 1.66 \\
\hline Bombas atómicas & 5.29 & 1.97 & Hitler & 4.68 & 2.28 \\
\hline Imperio Romano & 5.40 & 1.51 & Martín Lutero & 4.69 & 1.48 \\
\hline Atentado del 11 de septiembre & 5.49 & 1.70 & Fidel Castro & 4.70 & 1.76 \\
\hline Holocausto & 5.55 & 1.91 & Che Guevara & 4.96 & 1.78 \\
\hline Globalización & 5.59 & 1.41 & Alejandro Magno & 5.09 & 1.47 \\
\hline Revolución Francesa & 5.61 & 1.50 & Napoleón & 5.11 & 1.52 \\
\hline I Guerra Mundial & 5.63 & 1.60 & Juan Pablo II & 5.30 & 1.66 \\
\hline Desastres naturales & 5.64 & 1.63 & Cristóbal Colón & 5.51 & 1.61 \\
\hline Nacimiento de Jesús & 5.67 & 1.79 & Freud & 5.66 & 1.44 \\
\hline II Guerra Mundial & 5.84 & 1.60 & Aristóteles & 5.71 & 1.37 \\
\hline Caída Muro Berlín & 5.92 & 1.36 & Luther King & 5.75 & 1.43 \\
\hline Independencia colonias & 5.96 & 1.31 & Jesús & 5.79 & 1.67 \\
\hline Descubrimientos de América & 6.09 & 1.38 & Da Vinci & 5.83 & 1.38 \\
\hline Invención imprenta & 6.18 & 1.27 & Teresa de Calcuta & 5.94 & 1.39 \\
\hline Revolución Industrial & 6.21 & 1.13 & Mandela & 5.97 & 1.41 \\
\hline Evolución de la humanidad & 6.21 & 1.16 & Mahatma Gandhi & 6.10 & 1.17 \\
\hline Desarrollos tecnológicos & 6.22 & 1.10 & Einstein & 6.32 & 1.02 \\
\hline Era digital & 6.23 & 1.08 & & & \\
\hline Sistema democrático & 6.26 & 1.13 & & & \\
\hline $\begin{array}{l}\text { Descubrimientos } \\
\text { médicos/científicos }\end{array}$ & 6.63 & 0.78 & & & \\
\hline Abolición esclavitud & 6.72 & 0.80 & & & \\
\hline
\end{tabular}

En el dendograma de la figura 2 se observan con claridad 2 conglomerados. Uno, con eventos y personajes considerados más importantes (medias mayores a 4.84 para eventos y a 5.09 para personajes) que espacialmente se extiende desde la Primera Guerra Mundial hasta la Llegada del Hombre a la Luna, y otro, con eventos y personajes no tan importantes (medias entre 4.69 y 4.86 para los eventos y entre 3.71 y 4.96 para los personajes) que se extiende espacialmente desde Fidel Castro hasta Hitler. 


\section{pguerral}

sguerral

holocaustol

bombas!

guerrafrial

nazismol

torresgemelas!

irakl

desnaturales!

napoleonl

magnol

imperioromano

revfrancesal

globalizacionl

desamerical

colonl

revindustriall

imprental

esclavitudl

descientificos!

tecnologial

digitall

evolucionl

democracial

einteinl

gandhil

mandelal

davincil

kingl

independenciacolonias

muroberlinl

aristoteles!

freudl

calcutal

cristianismol

jesus!

papajpl

hombreluna

castrol

guevaral

revcubanal

stalinl

churchilll

mussolinil

revrusal

depresionl

luterol

kennedyl

bushl

thatcherl

binladenl

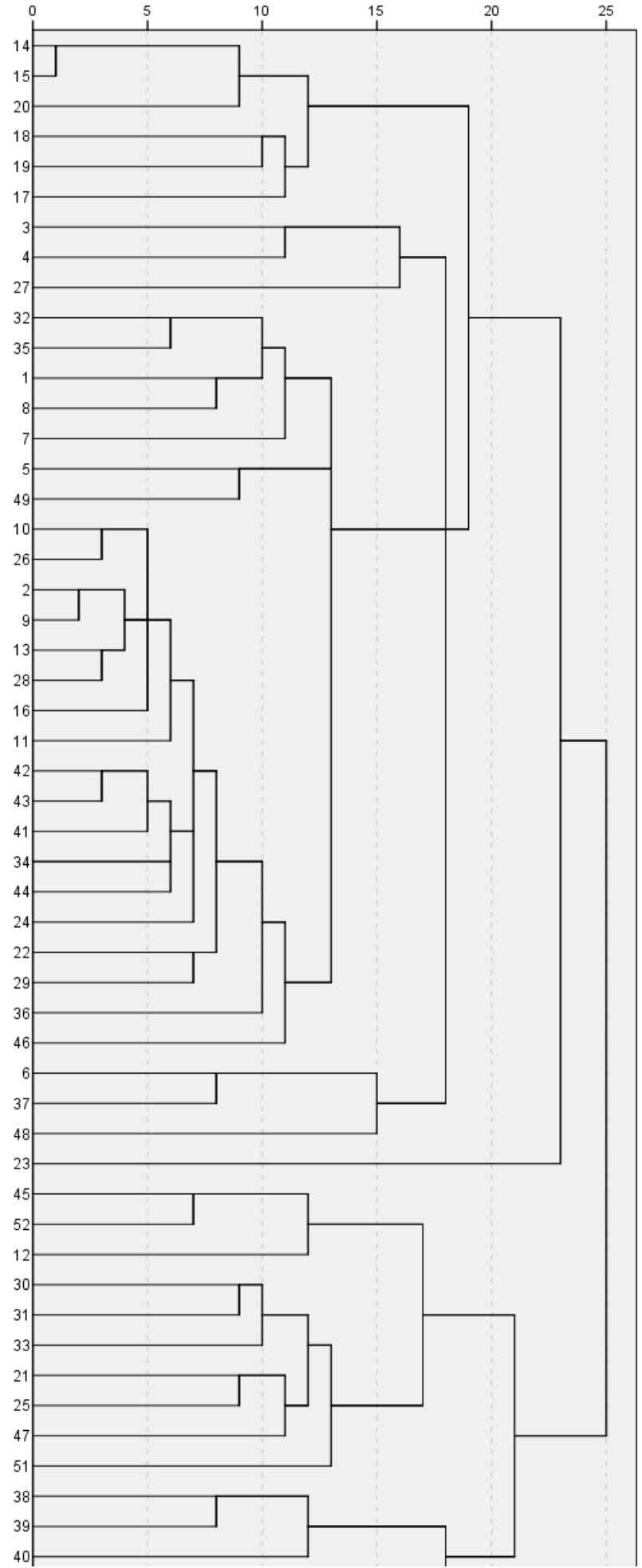

Figura 2. Dendrograma para importancia atribuida a los eventos y personajes presentados.

\section{Relación entre evaluación en dimensión positivo-negativo e importancia} atribuida a los eventos y personajes presentados

Con el fin de organizar los eventos y personajes en función de la evaluación subjetiva que evoca y la importancia atribuida, se confeccionó la tabla 3. La misma, construida en la forma de una tabla de contingencia, presenta nueve cuadrantes en los que los eventos y personajes se ubican en 
función de la media que obtienen en la evaluación en la dimensión positivonegativo y a la importancia que se le da. Así, la evaluación en la dimensión positivo-negativo presenta tres columnas: (a) negativo, eventos y personajes con puntuaciones entre 1 y 2.99; (b) intermedio, puntuaciones entre 3 y 4.99; y (c) positivo, puntuaciones entre 5 y 7 . Y la importancia atribuida exhibe tres filas: (a) bastante importancia, puntuaciones entre 3 y 4.99; (c) mucha importancia, puntuaciones entre 5 y 5.99; y (d) importancia extrema, puntuaciones entre 6 y 7.

Tabla 3.

Eventos y personajes de la historia universal según evaluación subjetiva e importancia.

\begin{tabular}{|c|c|c|c|c|c|}
\hline & & & EVALUACIÓN SUBJET & VA & \\
\hline & & $\begin{array}{c}\text { Negativo } \\
\text { (entre } 1 \text { y } 2.99 \text { ) }\end{array}$ & $\begin{array}{c}\text { Intermedio } \\
\text { (entre } 3 \text { y } 4.99 \text { ) }\end{array}$ & $\begin{array}{c}\text { Positivo } \\
\text { (entre } 5 \text { y } 7)\end{array}$ & TOTAL \\
\hline & $\begin{array}{r}\text { Bastante } \\
\text { (entre } 3 \mathrm{y} \\
4.99)\end{array}$ & $\begin{array}{c}\text { (9) Thatcher, Bush, } \\
\text { Guerra de Irak, } \\
\text { Guerra Fría, Gran } \\
\text { depresión, Hitler, Bin } \\
\text { Laden, Mussolini, } \\
\text { Stalin }\end{array}$ & $\begin{array}{c}\text { (8) Rev. Rusa, Rev. } \\
\text { Cubana, Fidel Castro, } \\
\text { Che Guevara, Llegada } \\
\text { hombre luna, Churchill, } \\
\text { Kennedy, Lutero }\end{array}$ & ---- & 17 \\
\hline 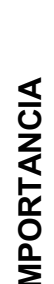 & $\begin{array}{r}\text { Mucha } \\
\text { (entre } 5 \mathrm{y} \\
5.99)\end{array}$ & $\begin{array}{c}\text { (7) I GM, II GM, } \\
\text { Holocausto, Nazismo, } \\
\text { bombas atómicas, } \\
\text { 11S, desastres } \\
\text { naturales }\end{array}$ & $\begin{array}{l}\text { (6) Imperio Romano, } \\
\text { Globalización, } \\
\text { Revolución Francesa, } \\
\text { Napoleón, Colón, } \\
\text { Alejandro Magno }\end{array}$ & $\begin{array}{c}\text { (11) Nacimiento Jesús, } \\
\text { Jesús de Nazaret, Caída } \\
\text { Muro Berlín, Indep. } \\
\text { colonias, Juan Pablo II, } \\
\text { Freud, Aristóteles, Luther } \\
\text { King, da Vinci, Mandela, } \\
\text { Madre Teresa }\end{array}$ & 24 \\
\hline & $\begin{array}{r}\text { Extrema } \\
(\text { entre } 6 \text { y } 7)\end{array}$ & ---- & $\begin{array}{c}\text { (1) Descubrimiento de } \\
\text { América }\end{array}$ & $\begin{array}{l}\text { (10) Rev. Ind., era digital, } \\
\text { evol. humanidad, } \\
\text { imprenta, desarrollos } \\
\text { tecnol., sist. democrático, } \\
\text { abolición esclavitud, } \\
\text { descub. } \\
\text { médicos/científicos, } \\
\text { Einstein, Gandhi }\end{array}$ & 11 \\
\hline & TOTAL & 16 & 15 & 21 & 52 \\
\hline
\end{tabular}

Nota: Los números indican cantidad de eventos/personajes.

Al analizar la distribución de frecuencias, se puede constatar que existe una asociación positiva entre la evaluación subjetiva evocada y el grado de importancia atribuido, $X^{2}(4, n=52)=23.68 ; p<.001$ (calculado mediante el Statistics Calculator 4.0 sobre la base de la distribución de frecuencias aquí presentada). Así, a los eventos y personajes considerados más negativos tiende a atribuírseles menor importancia, como por ejemplo el caso de George W. Bush y Margaret Thatcher, al tiempo que a los eventos y personajes más positivos se les asigna mucha importancia, como por ejemplo la Revolución Industrial, la era digital, la invención de la imprenta, la abolición de la esclavitud, Albert Einstein y Mahatma Gandhi. Si se efectúa este mismo análisis, pero para eventos y personajes por separado, se obtienen resultados 
similares (Eventos: $X^{2}(4, n=28)=15.83 ; p=.003$; Personajes: $X^{2}(4, n=24)=$ $17.13 ; p=.002)$. De hecho, al calcular el coeficiente de correlación para la relación entre evaluación en la dimensión positivo-negativo e importancia atribuida para eventos $(r=.38 ; n=299 ; p=.001)$ como para personajes $(r=$ $.59 ; n=325 ; p<.001)$, se verifica que a mejor evaluación del estímulo presentado (evento o personaje) más importancia se le atribuye.

\section{DISCUSIÓN}

El presente estudio buscó avanzar en las RSH siguiendo una línea que busca dar cuenta de una narrativa a través del anclaje valorativo y emocional de diferentes eventos y personajes universales (Liu et al., 2009), incorporando también en ella la lógica de héroes y villanos propuesta por Hanke et al. (2015), asi como los factores latentes de calamidades históricas, progreso histórico y resistencia a la opresión histórica identificados (Liu et al., 2012).

Todos los eventos y personajes evaluados por los participantes de forma negativa corresponden a la categoria de calamidades históricas (Liu et al., 2012) mientras que los eventos y personajes evaluados positivamente refieren al progreso histórico o a la resistencia contra la opresión. El efecto amortiguador de belicosidad de la Revolución Francesa por el sesgo nostálgico coincide con lo indicado por Páez et al. (2007) que, al explicar el sesgo de positividad hacia el pasado lejano, indican cómo eventos relativamente similares como la Revolución Francesa y las guerras mundiales muestran perfiles opuestos. Aun cuando los eventos produjeron centenares de miles de muertos, hay una visión globalmente favorable hacia la Revolución Francesa.

Dentro de los personajes evaluados de forma positiva, también pueden observarse tres personajes que, según Hanke et al. (2015), podian agruparse como "cristianos": la madre Teresa de Calcuta, Jesús de Nazaret y el papa Juan Pablo II. Es posible que a estos personajes también se les esté atribuyendo un significado ligado a la lucha contra la opresión, especialmente en lo que hace a la preocupación por los más pobres o vulnerables.

De esta manera, los eventos y personajes se ordenan bajo una lógica de calamidades versus progreso histórico-lucha contra la opresión, donde los personajes que son evaluados como villanos son aquellos relacionados con las calamidades (ej. Hitler con el Holocausto y el Nazismo; Bin Laden con el atentado del 11 de septiembre) y los personajes que despiertan evaluaciones subjetivas positivas son aquellos que se relacionan con el progreso (ej. Einstein, Da Vinci y Freud con descubrimientos médicos/científicos) y la resistencia a la opresión (ej. Gandhi, Mandela).

Esta lógica se confirma al observar un conglomerado -el más negativorelativo a las calamidades históricas, otro -el más positivo- relacionado con el progreso histórico y la lucha contra la opresión, y un tercer agrupamiento donde, si bien se incluyen eventos y personajes relacionados con el progreso histórico y la lucha contra la opresión, estos se relacionan más con luchas sociales y aparecen cuestiones relativas a conquistas y exploraciones.

El trabajo presenta como novedad en los estudios sobre RSH el análisis de la relación entre eventos y personajes. Respecto de las calamidades históricas, todas pertenecientes al último siglo, se confirmó la asociación a nivel representacional entre las bombas atómicas, el Holocausto, el nazismo y Hitler, entre las dos guerras mundiales y entre Mussolini, Bush Jr. y Thatcher. Esta última asociación resulta interesante dado que no se trata de 
personajes que hayan tenido relación entre sí, sino que más bien podría pensárselos como tres figuras que se opusieron o implementaron medidas contrarias a la lucha contra la opresión. En el conglomerado positivo, podría pensarse en la lucha cristiana contra la opresión (Jesús, el nacimiento del Cristianismo y el Papa Juan Pablo II) y en la predominancia del progreso histórico, que se visualiza en dos grupos de eventos: uno con sucesos concretos (Revolución Industrial, era digital y llegada del hombre a la Luna) y otro con eventos más generales (desarrollos tecnológicos y descubrimientos médicos-científicos). Las figuras que se agrupan pertenecen tanto al progreso histórico (da Vinci, Einstein) como a la lucha contra la opresión (Gandhi), confirmando que los temas más controversiales como la Revolución Cubana (con sus personajes: Fidel Castro y el Che Guevara) y el descubrimiento de América con su representante Cristóbal Colón, son los que suscitan mayor discrepancia en coherencia con lo hallado por Zubieta y Barreiro (2014), que observan que el Descubrimiento de América, aun como suceso de mucha importancia histórica, se transforma en "conquista", "civilización" o "evangelización" en función del posicionamiento ideológico o la religión de las personas. Aun así, Páez et al. (2007), al dar cuenta de la positividad del pasado lejano, resaltan también que la evaluación de los hechos más antiguos tiende a ser similar entre las naciones, como se verifica con el Descubrimiento de América, que recibe similares evaluaciones por parte de polacos, españoles, argentinos, chilenos, mexicanos y brasileros (Brasil \& Cabecinhas, 2017).

En lo que hace a la importancia atribuida a los eventos y personajes de la historia universal, esta es mayor en los eventos que en los personajes. Esto confirma la relevancia de los estímulos presentados en línea con lo hallado tanto por estudios previos transculturales (Hilton \& Liu, 2017; Liu \& Hilton, 2005; Liu et al., 2005; Liu et al., 2012; Hanke et al., 2015) como por aquellos realizados en el contexto argentino (Bombelli, Fernández, \& Sosa, 2013; Cejas et al., 2013). Asimismo, la relativa preeminencia general de los eventos sobre los personajes puede asociarse con lo apuntado por Hanke et al. (2015) en relación con su función diferencial para la cultura política de una nación: los eventos imparten lecciones mientras que los personajes transmiten valores.

Los eventos y personajes considerados más importantes se relacionaron con avances en los derechos del hombre y la libertad, el conocimiento y cambios en la vida social. Por su parte, los personajes menos importantes son aquellos que también provocaron una evaluación claramente negativa. De esta manera, los eventos y personajes se unifican a nivel temático a partir de las evaluaciones subjetivas que suscitan, pero se separan cuando se los examina en función de la importancia que se le otorga. Eventos como el Nazismo y el Holocausto cobran mayor relevancia en comparación con la figura de Hitler.

Siguiendo a Wertsch (1998), se pueden identificar distintos grupos temáticos, es decir, aquellos argumentos o significados que aparecen en un relato y que le dan un sentido unificado, que comprende tanto a las acciones como a las intenciones y motivos de los agentes históricos involucrados. Puede pensarse al totalitarismo como tema ordenador de los estímulos evaluados de forma más negativa: el Holocausto, el nazismo, Hitler, Stalin, las guerras, todos ellos fenómenos autoritarios que tuvieron lugar alrededor de la Segunda Guerra Mundial. Y, de forma más contemporánea, los atentados terroristas. Entre estos eventos y personajes totalitarios, se incluyen de forma interesante los desastres naturales, que pueden ser analizados como producto de la manipulación exagerada del hombre sobre la naturaleza. Entre los fenómenos 
evaluados de forma positiva, pueden distinguirse dos grupos, sin espacialidad histórica concreta. Uno, el valorado de forma más positiva, que da cuenta de progreso o avance de la humanidad, en lo que hace tanto a la ciencia (e.g. invención de la imprenta, descubrimientos tecnológicos, era digital, Einstein) como a los derechos del hombre (e.g. abolición de la esclavitud, democracia, Gandhi, madre Teresa de Calcuta, Luther King). Otro, con fenómenos relacionados a la cuestión americanista, más sociocéntrica en términos regionales como, por ejemplo: el descubrimiento de América y la Revolución Cubana.

En línea con lo observado por Techio et al. (2010), los hallazgos de este estudio dan fuerza a la idea de que las personas exhiben etnocentrismo en sus visiones de la historia y atribuyen importancia a los eventos y personajes relacionados con la historia de su pais o región. Esto sucede en el caso del descubrimiento de América, por remitir a un suceso fundamental para la identidad regional, pero en el caso de la Revolución Cubana, por la intima relación entre esta y una figura central para la misma, el Che Guevara. Es importante aclarar, respecto de este sociocentrismo, que en este relevamiento no se incluyó la Guerra de Malvinas por considerarse un hecho nacional, aunque, sin embargo, en distintos estudios realizados a nivel local (Bombelli et al., 2013; Fernández, Sosa, \& Zubieta, 2015) la Guerra de Malvinas aparece como un suceso importante de la historia universal. La profunda trascendencia de esta guerra en la memoria colectiva argentina probablemente también explique la evocación y alta valoración negativa que obtiene Margaret Thatcher, ícono del conflicto de Argentina con Inglaterra.

En el caso de la importancia atribuida a los distintos estímulos presentados, vuelve a aparecer el totalitarismo como ordenador, esta vez, de los fenómenos evaluados como relativamente menos importantes (e.g. Revolución Cubana, Revolución Rusa, Bush, Thatcher, Bin Laden, Hitler). Los encuentros violentos entre distintas culturas, como guerras, conquistas, ocupaciones o revueltas sociales se agruparon obteniendo una importancia media (e.g. Revolución Francesa, guerras mundiales, descubrimiento de América). Finalmente, eventos y personajes ligados a la temática de avance como los descubrimientos científicos y tecnológicos, y los fenómenos relacionados a los derechos humanos, son los evaluados como más importantes.

Los estímulos relacionados con la temática de avance o progreso son los evaluados de forma más positiva y a los cuales se les atribuye mayor importancia. Por el contrario, los fenómenos totalitarios son los evaluados de forma más negativa y con una importancia relativa más baja. Así, puede pensarse que la memoria colectiva se relaciona con el bienestar del grupo social, donde los eventos y personajes recordados tienden a ser positivos para mantener una buena imagen del grupo -aquí el grupo de referencia sería la humanidad- y, que cuando estos son negativos, se les tiende a atribuir menor importancia, a fin de lograr, a nivel de la memoria colectiva, una balanza positiva, afirmando que es cierto que existieron fenómenos negativos, pero estos tienen una importancia relativa menor a la de los eventos positivos.

La asociación entre evaluación en la dimensión negativo-positivo e importancia atribuida es más fuerte en el caso de los personajes que en el caso de los eventos, lo que puede basarse en una creencia general relativa a que, por un lado, las personas son más importantes que los eventos y, por el otro, que las personas buenas son más importantes que las personas malas. 
Queda pendiente para futuras investigaciones indagar en las RSH en función de los posicionamientos diferenciales que puedan surgir a partir de aspectos tales como valores y creencias, para ahondar sobre todo en la variabilidad obtenida en la evaluación de eventos y personajes como el descubrimiento de América y Colón, y la Revolución Cubana, el Che Guevara y Fidel Castro; y en la importancia atribuida a los eventos y personajes relacionados con las calamidades históricas.

En esta misma línea, al ser evidente la limitación que el tipo de muestra utilizada presenta, sería deseable poder avanzar en la incorporación de participantes que pueden no tener acceso a internet y dé muestras de otras regiones del país. Asimismo, otra línea de interés es la de encarar estudios comparativos a nivel regional sobre RSH de modo similar a lo que se está efectuando con otros estudios relativos a la $\mathrm{MC}$, por ejemplo, con relación al pasado reciente y las consecuencias psicológicas de la dictadura militar en distintos países de América Latina.

Además de las dificultades metodológicas que el instrumento puede presentar por trabajar con opciones de eventos y personajes dadas y no evocadas por los participantes, es clara también la limitación que acarrea desligar a los sucesos y figuras de su contexto tanto histórico como discursivo. En este sentido, es necesario considerar el análisis de la relación entre eventos y personajes con las concepciones de la historia y la historiografia del sentido común.

Dada la escasez de estudios previos locales que tomen a la historia desde una perspectiva psicosocial como representaciones constitutivas de la MC, el haber desarrollado un análisis de la evaluación e importancia atribuida a eventos y personajes es una contribución promisoria. En este sentido, se buscó avanzar, siguiendo la línea iniciada por Liu et al. (2005), en una dinámica de exploración que permite la comparación transcultural, a la vez que abre a nivel local una arista relevante de investigación sobre el sentido común y la historia.

\section{REFERENCIAS}

Bobowik, M., Páez, D., Liu, J., Espinosa, A., Techio, E., Zubieta, E., \& Cabecinhas, R. (2010). Beliefs about history, the meaning of historical events and culture of war. Revista de Psicologia, 28(1), 111-146.

Bombelli, J., Fernández, O., \& Sosa, F. (2013). Representaciones sociales de la historia argentina y género. Un estudio con estudiantes militares. Boletín de Psicología, 108, 71-89.

Bombelli, J., Sosa, F., Fernández, O., Cejas, L., \& Torres, J. (2013). Memoria colectiva: eventos relevantes de la historia universal para una muestra de población militar. Memorias de las Jornadas de Investigación, 20, 62-65.

Brasil, J. A. \& Cabecinhas, R. (2017). Social Representations of Latin American History and (Post) Colonial Relations in Brazil, Chile and Mexico. Journal of Social and Political Psychology, 5(2), 537-557. https://doi.org/10.5964/jspp.v5i2.701

Cejas, L., Fernández, O., \& Sosa, F. (2013, Septiembre). Memoria colectiva: personajes de la historia universal, un estudio con estudiantes de psicología. Ponencia presentada en III Congreso de Psicología del Tucumán de la Universidad Nacional de Tucumán, San Miguel de Tucumán, Argentina.

Fernández, O., Sosa, F., \& Zubieta, E. (2015). Memoria colectiva y posicionamientos diferenciales: figuras de la historia argentian en estudiantes de psicología. Anuario de Investigaciones, 22(1), 197-206.

Haas, V. \& Jodelet, D. (2000). La mémoire, ses aspects sociaux et collectives. En N. Roussiau, Psychologie sociale (pp. 121-134). Paris: Editions Inpress.

Halbwachs, M. (1925/2004). Los marcos sociales de la memoria. Caracas: Anthropos.

Halbwachs, M. (1968/1995). Memoria colectiva y memoria histórica. Revista Española de Investigaciones Sociológicas, 69, 209-219. https://doi.org/10.2307/40183784

Hanke, K., Liu, J. H., Sibley, C. G., Páez, D., Gaines, S. O., Maloney, G., . . Cabecinhas, R. (2015). "Heroes" and "Villains" of World History across Cultures. PLOS ONE, 1O(2), e0115641. https://doi.org/10.1371/journal.pone.0115641

Hilton, D. J. \& Liu, J. H. (2017). History as the narrative of a people: From function to structure and content. Memory Studies, 10(3), 297-309. https://doi.org/10.1177/1750698017701612

Laframboise, N. \& Acevedo, S. (2014, marzo). El hombre frente a la madre naturaleza. Finanzas \& 
Desarrollo, 3, 44-47.

Liu, J. H. \& Hilton, D. J. (2005). How the past weighs on the present: Social representations of history and their role in identity politics. British Journal of Social Psychology, 44(4), 537-556. https://doi.org/10.1348/014466605X27162

Liu, J. H., Hilton, D., Huang, L.-L., Gastardo-Conaco, C., Dresler-Hawke, E., Pittolo, F., . . . Kidaka, Y. (2005). Social representations of events and people in world history acrros 12 cultures. Journal of Cross-Cultural Psychology, 36(2), 171-191. https://doi.org/10.1177/0022022104272900

Liu, J. H., Páez, D., Hanke, K., Rosa, A., Hilton, D. J., Sibley, C. G., . . . Suwa, K. (2012). Cross-cultural dimensions of meaning in the evaluation of events in world history?: Perceptions of historical calamities and progress in cross-cultural data from thirty societies. Journal of Cross-Cultural Psychology, 43(2), 251-272. https://doi.org/10.1177/0022022110390926

Liu, J. H., Páez, D., Slawuta, P., Cabecinhas, R., Techio, E., Kokdemir, D., . . . Zlobina, A. (2009). Representing world history in the 21 st century: the impact of 9/11, the Iraq War, and the nation-state on dynamics of collective remenbering. Journal of Cross-Cultural Psychology, 40(4), 667-692. https://doi.org/10.1177/0022022109335557

Páez, D., Techio, E., Marques, J., \& Martín Beristain, C. (2007). Memoria colectiva y social. En J. F. Morales, M. C. Moya, E. Gaviria \& I. Cuadrado (Coords.), Psicología Social (pp. 693-716). Madrid: McGraw-Hill.

Páez, D., Valencia, J., Pannebaker, J., Rimé, B., \& Jodelet, D. (1998). Memoria colectiva de procesos politicos y culturales. Bilbao: Servicios Editoriales de la UPV.

Pennebaker, J., Páez, D., \& Deschamps, J. C. (2006). The social psychology of history: Defining the most important events of the last 10, 100, and 1000 years. Psicología Politica, 32, 15-32.

Rosa, A., Bellelli, G., \& Bakhurst, D. (Eds.) (2000). Memoria colectiva e identidad nacional. Madrid: Biblioteca Nueva.

Roth, J., Huber, M., Juenger, A., \& Liu, J. H. (2017). It's about valence: Historical continuity or historical discontinuity as a threat to social identity. Journal of Social and Political Psychology, 5(2), 320-341. https://doi.org/10.5964/jspp.v5i2.677

Sibley, C. S., Liu, J. H., Duckitt, J., \& Khan, S. S. (2008). Social representations of history and the legitimation of social inequality: The form and function of historical negation. European Journal of Psychology, 38, 542-565. https://doi.org/10.1002/ejsp.449

Sosa, F., Bombelli, J. I., Cejas, L., \& Fernández, O. (2013, Septiembre). Representaciones sociales de la historia universal: figuras relevantes de la historia en estudiantes de formación universitaria militar. Ponencia presentada en III Congreso de Psicología del Tucumán de la Universidad Nacional de Tucumán, San Miguel de Tucumán, Argentina.

Sosa, F., Natapof, D., \& Zubieta, E. (2017). Representaciones Sociales de la Segunda Guerra Mundial: Valores y Dominancia social. Psicodebate, 17(2), 55-68. https://doi.org/10.18682/pd.v17i2.694

Tajfel, H. \& Turner, J. C. (2004). The social identity theory of intergroup behavior. En J. T. Jost \& J. Sidanius (Eds.), Key readings in social psychology. Political psychology: Key readings (pp. 276-293). New York: Psychology Press. https://doi.org/10.4324/9780203505984-16

Techio, E., Bobowik, M., Páez, D., Cabecinhas, R., Liu, J., Zubieta, E. \& Espinosa, A. (2010). Social representations of history, wars and politics in Latin America, Europe and Africa. Revista de Psicología Social, 25(1), 11-26. https://doi.org/10.1174/021347410790193441

Valencia, J. F. \& Páez, D. (1999). Generación, polémica pública, clima social y recuerdo de hechos políticos. Psicología Política, 18, 11-30.

Valencia, J. F., Momoitio, J., \& Idayaga, N. (2010). Social representations and memory: The psychosocial impact of the Spanish "Law of Memory", related to the Spanish Civil War. Revista de Psicología Social, 25(1), 73-86. https://doi.org/10.1174/021347410790193496

Wagner, W. (2015). Representation in action. En G. Sammut, E. Andreouli, G. Gaskell \& J. Valsiner (Eds.), The Cambridge Handbook of Social Representations (pp. 12-28). Cambridge, UK: Cambridge University Press. https://doi.org/10.1017/CBO9781107323650.004

Wertsch, J. (1998). La mente en acción. Buenos Aires: Aique.

Zaromb, F. M., Liu, J. H., Páez, D., Hanke, K., Putnam, A. L., \& Roediger III, H. L. (2018). We made history: Citizens of 35 countries overestimate their Nation's role in world history. Journal of Applied Research in Memory and Cognition, 7(4), 521-528. https://doi.org/10.1016/j.jarmac.2018.05.006

Zubieta, E. \& Barreiro, A. V. (2014). Memoria colectiva y representaciones sociales de la historia. Estudio preliminar con estudiantes universitarios argentinos. En Zubieta, E., J. F. Valencia, \& Delfino, G., Psicología social y politica: procesos teóricos y estudios aplicados (pp. 589-610). Buenos Aires: Eudeba.

Recibido 16-09-2019 | Aceptado 08-04-2020

Este trabajo se encuentra bajo una Licencia Creative Commons Atribución 4.0 Internacional que permite a terceros utilizar lo publicado siempre que se dé el crédito pertinente a los autores y a Psicodebate 